\title{
Punção venosa periférica difícil: revisão integrativa
}

\author{
Difficult peripheral venous puncture in adults: integrative review \\ Punción venosa periférica difícil: revisión integradora
}

\begin{abstract}
Andressa Moreira Marinho'; Fabiano Henrique Oliveira Sabino"; Damiana Aparecida Trindade Monteiro "I"; Viviane da Silva Alves Filgueira ${ }^{\prime V}$; Guilherme Nascimento de Azevedo ${ }^{\text {; }}$ Silmara Elaine Malaguti Toffano ${ }^{\mathrm{VI}}$
\end{abstract}

\begin{abstract}
RESUMO
Objetivo: identificar os fatores que dificultam a punção venosa periférica em adultos. Método: revisão integrativa da literatura, sem recorte temporal. A busca dos artigos ocorreu no mês de janeiro de 2018 nas seguintes bases de dados: PubMed, LILACS, CINAHL, SciELO, Web of Science e Scopus, com inclusão de 12 artigos. Foram analisados quanto ao ano, país de publicação, fatores associados e nível de evidência. Resultados: foram encontradas publicações de 2003 a 2017. A prevalência da punção venosa periférica difícil variou entre $17 \%$ e $59,3 \%$. Entre os fatores que dificultam a punção, os mais frequentes foram: demográficos (sexo, idade), clínicos (comorbidades, estado nutricional, visibilidade e palpabilidade da rede venosa), do dispositivo (calibre e modelo) e a habilidade do profissional. Conclusão: a dificuldade para punção é variável de acordo com a complexidade assistencial. Fatores demográficos e clínicos, do dispositivo e a habilidade do profissional devem ser considerados. Descritores: Cateterismo periférico; enfermagem; adulto; dificuldades.
\end{abstract}

\section{ABSTRACT}

Objective: to identify factors that pose difficulties in peripheral venipuncture in adults. Method: in this integrative literature review, with no time cutoff, search of the PubMed, LILACS, CINAHL, SciELO, Web of Science, and Scopus databases, in January 2018, yielded 12 articles for inclusion. These were examined for year, country of publication, associated factors, and level of evidence. Results: publications were found from 2003 to 2017. The prevalence of difficult peripheral venipuncture ranged from $17 \%$ to $59.3 \%$. The most frequent factors making for difficult puncture were: demographic (gender, age), clinical (comorbidities, nutritional status, visibility and palpability of the venous network), device-related (caliber and model), and professional skill. Conclusion: difficulty in puncture varies with complexity of care. Demographic-, clinical-, device- and professional skill-related factors should be considered.

Descriptors: Catheterization, peripheral; nursing; adult; difficulties.

\section{RESUMEN}

Objetivo: identificar los factores que dificultan la punción venosa periférica en adultos. Método: revisión integradora de la literatura, sin recorte temporal. Los artículos se buscaron en enero de 2018 en las siguientes bases de datos: PubMed, LILACS, CINAHL, SciELO, Web of Science y Scopus, con inclusión de 12 artículos. El análisis se hizo respecto al año, al país de publicación, a los factores asociados y al nivel de evidencia. Resultados: se encontraron publicaciones entre 2003 y 2017. La prevalencia de punción venosa periférica difícil osciló entre un $17 \%$ y un $59.3 \%$. Entre los factores que dificultan la punción, los más frecuentes fueron: demográficos (género, edad), clínicos (comorbilidades, estado nutricional, visibilidad y cuán palpable es la red venosa), el dispositivo (calibre y modelo) y la habilidad del profesional. Conclusión: la dificultad en la punción varía según la complejidad de la atención. Deben considerarse factores demográficos y clínicos, del dispositivo y la habilidad del profesional.

Descriptores: Cateterismo periférico; enfermería; adulto; dificultades.

\section{INTRODUÇÃO}

A punção venosa periférica (PVP) é um procedimento no âmbito hospitalar ${ }^{1}$, e mais da metade dos pacientes admitidos necessitam da inserção de um cateter venoso periférico (CVP) ${ }^{2}$.

$\mathrm{O}$ sucesso na primeira tentativa de PVP além de reduzir desconfortos ao paciente reduz custos com materiais ${ }^{3} \mathrm{e}$ proporciona início da terapia estabelecida ${ }^{4}$. Entretanto um a cada nove ou dez adultos, e que necessitam de um CVP, apresentam dificuldade para punção venosa 5 .

Pesquisadores consideram punção venosa periférica difícil (PVPD) quando há mais de uma tentativa de inserção $0^{5,6}$ ou quando há mais de duas ou três tentativas. 
Alguns fatores podem contribuir para PVPD entre eles: a idade ${ }^{5,8,9}$, a cor da pele $\mathrm{e}^{5}$, o estado nutricional ${ }^{7,8}$, a complexidade clínica do paciente $^{8}$, a história de $\mathrm{PVP}^{5,8}$, o uso de quimioterapia ${ }^{5,7,8}$, o tipo e calibre do CVP $^{9,10}$, que podem influenciar no aumento do número de tentativas de inserção e ainda podem afetar a segurança do paciente no ambiente hospitalar ${ }^{5,7-10}$.

Por se tratar de uma temática inovadora, e que representa a prática da enfermagem no ambiente hospitalar, se faz necessária a consolidação de pesquisa e estudos que apoiam a prática baseada em evidências e a segurança do paciente.

Diante do exposto, este estudo teve como questão norteadora: Quais os fatores que dificultam a punção venosa periférica em adultos? Sendo o objetivo, identificar os fatores que dificultam a punção venosa periférica em adultos.

\section{METOdOLOGIA}

Trata-se de um estudo primário, de revisão da literatura. A revisão integrativa é um método que sintetiza a literatura quanto a determinado problema clínico ou fenômeno de interesse incorporando múltiplas perspectivas e tipos de literatura ${ }^{11}$.

Metodologia que contribui para o processo de sistematização e análise dos resultados, visando à compreensão de determinado tema, a partir de outros estudos independentes ${ }^{12}$. A diversidade do sistema de amostragem é a principal característica deste método de revisão. Assim, o revisor pode incluir estudos com diferentes delineamentos de pesquisa $^{11}$.

Foi adotado para o delineamento da busca a estratégia PICO. O acrônimo PICO representa Paciente, Intervenção, Comparação e Outcomes (desfecho) ${ }^{13}$.

Diante da questão de investigação, a estratégia PICO foi representada por: P - População: Adulto; I - Intervenção: Cateterismo Periférico; C - Controle: não possui e O - Desfecho: Dificuldade.

O estudo foi realizado seguindo as seis etapas preconizadas: definição da hipótese ou questão norteadora (definição do tema e pergunta de investigação, seleção dos descritores); busca na literatura (base de dados, desenvolvimento da estratégia, busca nas bases de dados e seleção dos estudos); categorização das informações (sumarização e formação do banco de dados); avaliação dos estudos incluídos na revisão (análise crítica e inclusão e exclusão); interpretação dos resultados e síntese do conhecimento ${ }^{11}$.

A busca dos artigos ocorreu no mês de janeiro de 2018, nas seguintes bases de dados: National Library of Medicine (PubMed), Literatura Latino-americana e do Caribe em Ciências da Saúde (LILACS), Cummulative Index to Nursing and Allied Health Literature (CINAHL), Scientific Electronic Library Online (SciELO), Web of Science e Scopus. O cruzamento ocorreu por meio dos descritores controlados Medical Subject Headings (MeSH) e Descritores em Ciências da Saúde (DeCS) utilizando as seguintes palavras-chave: adults AND catheterization; peripheral AND difficult e adulto AND cateterismo OR punção venosa AND difícil OR dificuldade.

Três revisores independentes realizaram a busca e seleção dos artigos. Os critérios de inclusão foram: publicações cuja temática estivesse relacionada à PVP, independente do período, idioma da publicação e que sejam realizados com sujeitos adultos com CVP. Foram excluídos estudos que utilizaram tecnologias como ultrassom e em ambiente simulado (manequins ou simuladores de PVP) além de revisões, monografias, dissertações ou teses.

A primeira seleção foi realizada por meio de leitura dos títulos e resumos, destacando os que atendiam aos critérios e respondiam a questão norteadora. Nesta etapa, foram excluídos os que possuíam títulos repetidos e os que não se encaixavam na temática proposta.

Em seguida, os artigos foram minuciosamente lidos na integra e traduzidos. A partir dessa etapa, foram retirados os artigos que não contemplavam os critérios.

Para a análise dos estudos e síntese das informações, foi utilizado o instrumento validado ${ }^{14}$ de modo a sistematizar os resultados, sendo organizadas por planilhas no Microsoft Office ${ }^{\circledR}$ do Excel ${ }^{\circledR}$, de acordo com a identificação dos estudos, área de publicação, características metodológicas e avaliação do rigor metodológico, inclusive o nível de evidência ${ }^{15}$.

Foram considerados os resultados identificados na literatura, como variáveis profissionais (tempo de experiência e categoria profissional), clínicos (comorbidades, estado nutricional, visibilidade e palpabilidade da rede venosa), do dispositivo (calibre e modelo) e a habilidade do profissional. 


\section{RESULTADOS}

Ao todo foram identificados 547 estudos. Sendo que, na base de dados PubMed foram encontrados $286(52,3 \%)$, na base Scopus $200(36,6 \%)$, EBSCO $33(6 \%)$, Web of Science foram encontrados $23(4,2 \%)$, LILACS $4(0,7 \%)$ e na SciELO apenas 1 estudo (0,18\%).

Permaneceram para análise 93 estudos. Dentre os 454 estudos descartados, 64 estavam repetidos e 390 não cabiam na temática ou não contemplavam os critérios de elegibilidade. Na segunda seleção, após leitura minuciosa dos textos, chegou-se ao número de 12 estudos, conforme mostra a Figura 1.

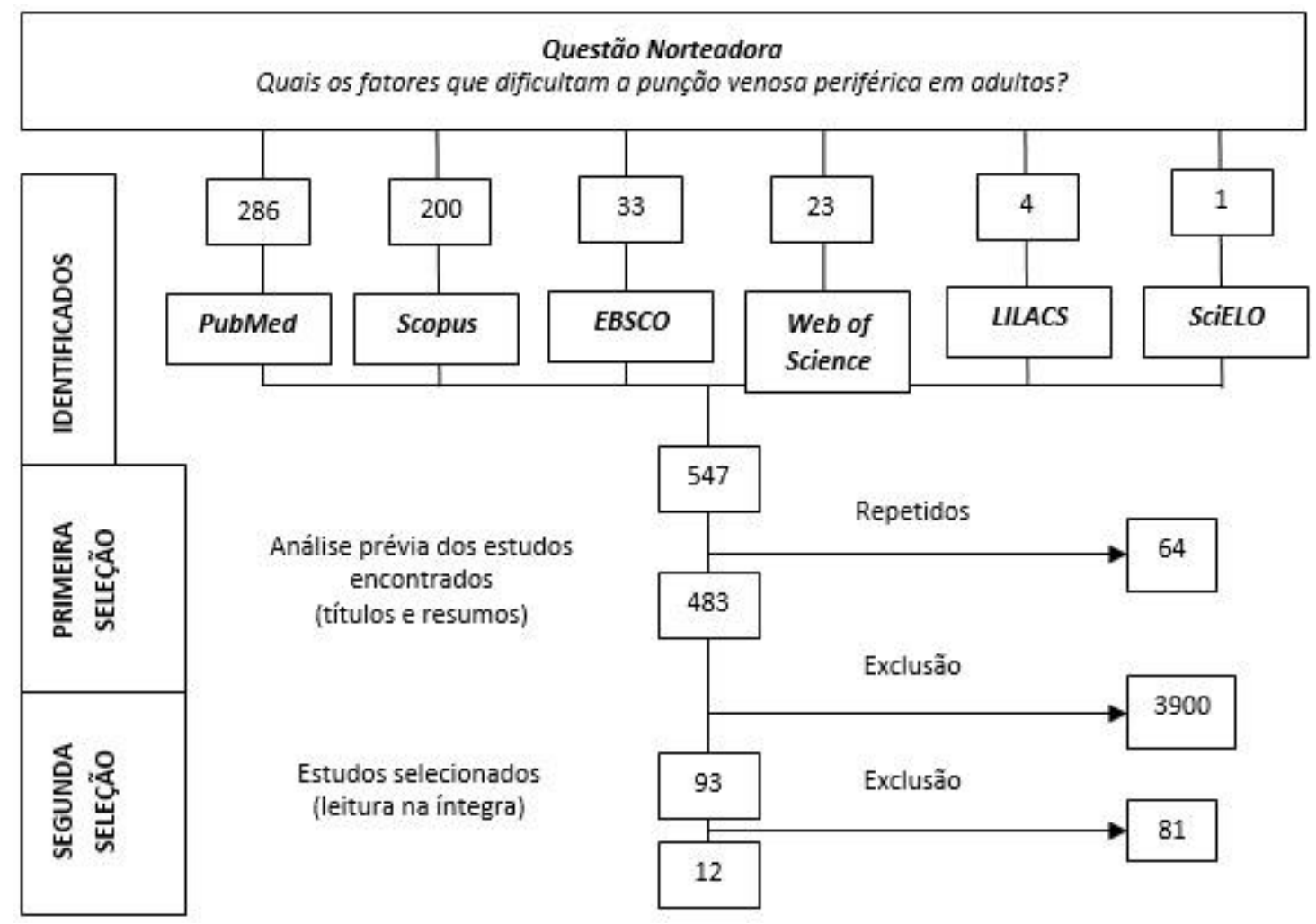

FIGURA 1: Fluxograma da busca, seleção e exclusão dos estudos. Uberaba, MG, Brasil, 2018.

Foram encontradas publicações de 2003 a 2017 3,5,6,8,9,16-22, segundo a Figura 2. Os países de origem dos estudos foram Austrália, Espanha, Itália e Holanda com um estudo em cada país, totalizando 4 (33,3\%); França e EUA com quatro estudos cada, perfazendo 8 (66,7\%).

Foi identificado um aumento do número de publicações realizadas nos últimos anos, principalmente em 2016 com 3 (25\%) artigos, 2014 e 2017 com dois textos cada, somando 4 (33,3\%). Os outros anos apresentaram apenas um artigo, reunindo 5 (41,6\%). Não foram encontradas publicações brasileiras. Apenas um estudo apresentava abordagem prospectiva e randomizado, sendo classificado como nível de evidência 2B, os demais foram todos observacionais. Ver Figura 3.

Quanto aos fatores demográficos e clínicos foram indicados: sexo ${ }^{8,9}$, cor da pele , idade $^{3,8,9,16}$, obesidade ${ }^{9,17,18}$ ou desnutrição ${ }^{6,18}$, higiene do paciente ${ }^{19}$, complexidade clínica ${ }^{6}$, desidratação ${ }^{3,18}$, visibilidade e/ou palpabilidade da veia $^{3,9,18,20}$, doenças crônicas ${ }^{5,8,18}$ e histórico de PVPD ${ }^{3,9,20}$, entre outras condições.

Em relação aos fatores associados ao dispositivo foram apontados: calibre do cateter ${ }^{21}$ e o tipo do dispositivo ${ }^{22}$. Ainda, a experiência e a habilidade do profissional que realiza a PVP foram indicadas $6,19,22$. 


\begin{tabular}{|c|c|c|}
\hline Título do artigo & País & $\begin{array}{c}\text { Ano de } \\
\text { publicação }\end{array}$ \\
\hline Is peripheral line placement more difficult in obese than in lean patients? ${ }^{17}$ & França & 2003 \\
\hline $\begin{array}{l}\text { Variables influencing intravenous catheter insertion difficulty and failure: an } \\
\text { analysis of } 339 \text { intravenous catheter insertions }{ }^{21}\end{array}$ & Estados Unidos da América & 2005 \\
\hline $\begin{array}{l}\text { Prospective evaluation of peripheral venous access difficulty in emergency } \\
\text { care }^{19}\end{array}$ & França & 2007 \\
\hline A prospective randomized trial of two safety peripheral intravenous catheters ${ }^{22}$ & França & 2008 \\
\hline $\begin{array}{l}\text { Predicting peripheral venous access difficulty in the emergency department } \\
\text { using body mass index and a clinical evaluation of venous accessibility }\end{array}$ & França & 2012 \\
\hline Risk factors associated with difficult venous access in adult ED patients ${ }^{5}$ & Estados Unidos da América & 2014 \\
\hline $\begin{array}{l}\text { Association between multiple IV attempts and perceived pain levels in the } \\
\text { emergency department }{ }^{16}\end{array}$ & Estados Unidos da América & 2014 \\
\hline $\begin{array}{l}\text { Development of the A-DIVA Scale: a clinical predictive scale to identify difficult } \\
\text { intravenous access in adult patients based on clinical observations }{ }^{20}\end{array}$ & Holanda & 2016 \\
\hline $\begin{array}{l}\text { Predicting and preventing peripheral intravenous cannula insertion failure in } \\
\text { the emergency department: clinician 'gestalt' wins again }{ }^{18}\end{array}$ & Austrália & 2016 \\
\hline $\begin{array}{l}\text { Factors affecting difficult peripheral intravenous cannulation in adults: a } \\
\text { prospective observational study }{ }^{9}\end{array}$ & Itália & 2016 \\
\hline $\begin{array}{l}\text { Prevalence of difficult venous access and associated risk factors in highly } \\
\text { complex hospitalised patients }{ }^{8}\end{array}$ & Espanha & 2017 \\
\hline $\begin{array}{l}\text { Establishing a dedicated difficult vascular access team in the emergency } \\
\text { department }^{3}\end{array}$ & Estados Unidos da América & 2017 \\
\hline
\end{tabular}

FIGURA 2: Síntese dos artigos incluídos por, título, país e ano de publicação. Uberaba, 2018.

\begin{tabular}{|c|c|c|c|}
\hline Estudo & $\begin{array}{l}\text { Nível de } \\
\text { Evidência }\end{array}$ & Delineamento & Fatores associados à punção venosa difícil em adultos \\
\hline $\mathrm{E}^{17}$ & $2 \mathrm{C}$ & Observacional & Obesidade \\
\hline $\mathrm{E}^{21}$ & $2 \mathrm{C}$ & Observacional & Experiência profissional \\
\hline $\mathrm{E}^{19}$ & $2 \mathrm{C}$ & Observacional & Calibre do cateter; habilidade do profissional; higiene do paciente \\
\hline$E^{22}$ & $2 \mathrm{~B}$ & $\begin{array}{l}\text { Prospectivo e } \\
\text { randomizado }\end{array}$ & Tipo de dispositivo/cateter \\
\hline $\mathrm{E}^{6}$ & $2 \mathrm{C}$ & Observacional & Complexidade clínica; habilidade do profissional; índice de massa corporal $<18,5$ \\
\hline$E^{5}$ & $2 \mathrm{C}$ & Observacional & Diabetes, doença falciforme e abuso de drogas endovenosas \\
\hline $\mathrm{E}^{16}$ & $2 \mathrm{C}$ & Observacional & Idade \\
\hline $\mathrm{E}^{20}$ & $2 \mathrm{C}$ & Observacional & Veia palpável ou visível, de menor calibre; história de PVPD; cirurgia não eletiva \\
\hline $\mathrm{E}^{18}$ & $2 \mathrm{C}$ & Observacional & $\begin{array}{l}\text { Calibre da veia; veia palpável; internação prévia; abuso de drogas endovenosas; } \\
\text { obesidade ou caquexia; quimioterapia recente, anormalidade da pele, doença } \\
\text { crônica, desidratação, agitação e instabilidade hemodinâmica. }\end{array}$ \\
\hline $\mathrm{E}^{9}$ & $2 \mathrm{C}$ & Observacional & $\begin{array}{l}\text { Veia palpável ou visível, idade, quimioterapia, sexo, fragilidade vascular, historia de } \\
\text { PVPD, válvulas, obesidade }\end{array}$ \\
\hline $\mathrm{E}^{8}$ & $2 \mathrm{C}$ & Observacional & $\begin{array}{l}\text { Sexo feminino; doença osteoarticular; idade; história de tratamento com } \\
\text { anticoagulantes ou corticosteróides; }\end{array}$ \\
\hline$E^{3}$ & $2 \mathrm{C}$ & Observacional & $\begin{array}{l}\text { Idade, desidratação, cor da pele, história de PVPD, tratamentos ou cirurgia prévias, } \\
\text { veias não visíveis }\end{array}$ \\
\hline
\end{tabular}

FIGURA 3: Síntese dos artigos incluídos artigo, nível de evidência, delineamento e fatores associados à punção venosa difícil em adultos. Uberaba, 2018.

\section{DISCUSSÃO}

A prevalência da PVPD é variável, contudo estudos indicam valores entre $17 \%^{5}$ até $59,3 \%{ }^{8}$.

Foi verificado um predomínio de estudos observacionais, com nível de evidência $2 \mathrm{C}$ que abordaram os fatores associados de modo simultâneo, não sendo possível isolar as variáveis e verificar sua associação com a PVPD.

A presença de comorbidades foi indicada como fator a ser considerado. Estudo brasileiro associou a presença de doença crônica não transmissível com o aumento de internação ${ }^{23}$. 
A cada processo de hospitalização o indivíduo é submetido a diferentes procedimentos terapêuticos e diagnósticos, no entanto, tais procedimentos apresentam em sua maioria a necessidade de um acesso venoso, comprometendo a rede vascular.

Foi destacado pelos artigos que a visibilidade e a palpabilidade da veia são consideradas cruciais no sucesso da PVP $3,7,9,18,20,24$

As tecnologias que auxiliam a visibilidade da veia, como a utilização do ultrassom, têm contribuído para a taxa de sucesso da punção, inclusive para adultos com PVPD ${ }^{20}$.

Pesquisadores apontam que, incorporando o uso do ultrassom, a taxa de sucesso na primeira tentativa de PVP foi de $64,9 \%$ e que, ainda, foi possível a punção de veias mais profundas e de maior calibre ${ }^{25}$.

Entretanto, a tecnologia apenas não é suficiente para o sucesso no procedimento. Assim como apresentado em estudos $^{6,7,19,22}$, o profissional, ao executar o procedimento, necessita de conhecimentos e habilidades.

A seleção de profissionais com experiência em PVP para procedimentos em pacientes previamente identificados como de difícil acesso é recomendada, uma vez que equipes especializadas em punções difíceis minimizam os custos com materiais e recursos humanos e, aumentam a satisfação do paciente ${ }^{3,26}$. Outra estratégia necessária é o estabelecimento de protocolos institucionais ${ }^{27}$, treinamentos e materiais educativos ${ }^{28}$ visando à redução das múltiplas tentativas de punção.

A terapia infusional, embora frequentemente presente na prática clínica e no cotidiano da enfermagem, carece de melhorias no cuidado. A vinculação das práticas assistenciais-acadêmicas, e a incorporação de novos dispositivos e tecnologias são apresentadas como propostas ${ }^{29,30}$.

A exclusão de artigos que não estavam disponíveis gratuitamente foi considerada uma limitação do estudo.

\section{CONCLUSÃO}

A ocorrência de PVPD foi associada a fatores demográficos (sexo, idade), clínicos (comorbidades, estado nutricional, visibilidade e palpabilidade da rede venosa), do dispositivo (calibre e modelo) e à habilidade do profissional. Apenas um estudo não foi observacional sendo necessária a realização de estudos com outros desenhos e abordagens com melhores níveis de evidencias.

Identificar os fatores associados à PVPD poderá nortear a conduta de profissionais, assim como contribuir para o desenvolvimento de ferramentas de rastreio e estratégias para minimizar múltiplas tentativas ou insucesso da PVP. O uso de tecnologias como o ultrassom vem auxiliando e reduzindo as taxas de falhas, portanto, é válido para o enfermeiro conhecer e fazer uso das mesmas, pois, uma inserção com sucesso já na sua primeira tentativa reduz o desconforto para o paciente, e consequentemente, diminui a frustração do profissional e dos custos para a instituição.

Para uma melhor compreensão da PVPD, é de suma importância a realização de outros estudos acerca da temática, principalmente sobre os fatores demográficos e clínicos, que são os prevalentes.

\section{REFERÊNCIAS}

1. Institute for Safe Medication Practices (ISMP). ISMP Safe Practice Guidelines for Adult IV Push Medications; 2015 [cited 2019 Feb 10]. Available from: https://www.ismp.org/guidelines/iv-push

2. Alexandrou E, Ray-Barruel G, Carr PJ, Frost S, Inwood S, Higgings N, et al. International prevalence of the use of peripheral intravenous catheters. J. Hosp. Med. 2015[cited 2019 Feb 10]; 10(8):530-3. DOI: https://doi.org/10.1002/jhm.2389

3. Whalen M, Maliszewski B, Baptiste DL. Establishing a dedicated difficult vascular access team in the emergency department: a needs assessment. J. Infus. Nurs. 2017 [cited 2019 Feb 21]; 40(3):149-54. DOI: https://doi.org/10.1097/NAN.0000000000000218

4. Witting, MD. IV access difficulty: incidence and delays in an urban emergency department. J. Emerg. Med. 2012 [cited 2019 Feb 09]; 42(4):483-7. DOI: https://doi.org/10.1016/j.jemermed.2011.07.030

5. Fields, JM, Piela NE, Au AK, Ku BS. Risk factors associated with difficult venous access in adult ED patients. Am. J. Emerg. Med. 2014 [cited 2019 Feb 17]; 32(10):1179-82. DOI: https://doi.org/10.1016/j.ajem.2014.07.008

6. Sebbane M, Claret PG, Lefebvre S, Mercier G, Rubenovitch J, Jreige R. Predicting peripheral venous access difficulty in the emergency department using body mass index and a clinical evaluation of venous accessibility. J. Emerg. Med. 2012 [cited 2019 Feb 21]; 44(2): 299-305. DOI: https://doi.org/10.1016/j.jemermed.2012.07.051

7. Carr PJ, Rippey JC, Budgeon CA, Cooke ML, Higgins N, Rickard CM. Insertion of peripheral intravenous cannula in the emergency department: factors associated with first-time insertion success. J. Vasc. Access. 2016 [cited 2019 Feb 21]; 17(2):182-90. DOI: https://doi.org/10.5301/jva.5000487

8. Armenteros-Yeguas V, Gárate-Echenique L, Tomáz-López MA, Cristóbal-Domínguez E, Moreno-de Gusmão B, Miranda-Serrano $\mathrm{E}$, et al. Prevalence of difficult venous access and associated risk factors in highly complex hospitalized patients. J. Clin. Nurs. 2017 [cited 2019 Feb 18]; 26(23-24):4267-45. DOI: https://doi.org/10.1111/jocn.13750 
9. Piredda M, Biagioli V, Barella B, Carpisassi I, Ghinelli R, Giannarelli D, et al. Factors affecting difficult peripheral intravenous cannulation in adults: a prospective observational study. J. Clin. Nurs. 2017 [cited 2019 Feb 21]; 26(7-8):1074-84. DOI: https://doi.org/10.1111/jocn.13444

10. Malyon L, Ullman AJ, Phillips N, Young J, Kleidon T, Murfield J, et al. Peripheral intravenous catheter duration and failure in paediatric acute care: a prospective cohort study. Emerg. Med. Australas. 2014 [cited 2019 Feb 17]; 26(6):602-8. DOI: https://doi.org/10.1111/1742-6723.12305

11. Mendes KDS, Silveira RCCP, Galvão CM. Integrative literature review: a research method to incorporate evidence in health care and nursing. Texto \& contexto enferm. (Online). 2008 [cited 2019 Feb 21]; 17(4):758-64. DOI: http://dx.doi.org/10.1590/S010407072008000400018

12. Lanzoni GMM, Meirelles BHS. Leadership of the Nurse: an Integrative Literature Review. Rev. latinoam. enferm. (Online). 2011[cited 2019 Feb 21];19(3): 651-8. DOI: http://dx.doi.org/10.1590/S0104-11692011000300026

13. Santos CMC, Pimenta CAM, Nobre MRC. The pico strategy for the research question construction and evidence search. Rev. latinoam. enferm. (Online). 2007 [cited 2019 Fev 18]; 15(3). DOI: http://dx.doi.org/10.1590/S0104-11692007000300023

14. Ursi ES, Galvão CM. Perioperative prevention of skin injury: an integrative literature review. Rev. latinoam. enferm. (Online). 2006 [cited 2019 Feb 15];14(1):124-31. DOI: http://dx.doi.org/10.1590/S0104-11692006000100017

15. Pedrosa KKA, Oliveira ICM, Feijão AR, Machado RC. Evidence-based nursing: characteristics of studies in brazil. Cogitare enferm. 2015 [cited 2019 Feb 21]; 20(4)733-41. DOI: http://dx.doi.org/10.5380/ce.v20i4.40768

16. Fields JM, Piela NE, Ku BS. Association between multiple IV attempts and perceived pain levels in the emergency department. J. Vasc. Access. 2014 [cited 2019 Feb 18]; 15(6):514-8. DOI: https://doi.org/10.5301/jva.5000282

17. Juvin P, Blarel A, Bruno F, Desmonts JM. Is peripheral line placement more difficult in obese than in lean patients? Anesth. Analg. 2003 [cited 2019 Feb 21]; 96(4):1218. Available from: https://www.ncbi.nlm.nih.gov/pubmed/12651688

18. Rippey JC, Carr PJ, Cooke M, Higgins N, Rickard CM. Predicting and preventing peripheral intravenous cannula insertion failure in the emergency department: clinician 'gestalt' wins again. Emerg. Med. Australas. 2016 [cited 2019 Feb 21]; 28(6):658-65. DOI: https://doi.org/10.1111/1742-6723.12695

19. Lapostolle F, Catineau J, Garrigue B, Monmarteau V, Houssaye T, Vecci I, et al. Prospective evaluation of peripheral venous access difficulty in emergency care. Intensive Care Med. 2007 [cited 2019 Feb 21]; 33(8):1452-7. Available from: https://www.ncbi.nlm.nih.gov/pubmed/17554524

20. Van Loon FH, Puijn LA, Houterman S, Bouwman AR. Development of the A-DIVA Scale: a clinical predictive scale to identify difficult intravenous access in adult patients based on clinical observations. Medicine (Baltimore). 2016 [cited 2019 Feb 21]; 95(16):e3428. DOI: https://doi.org/10.1097/MD.0000000000003428

21. Jacobson AF, Winslow EH. Variables influencing intravenous catheter insertion difficulty and failure: An analysis of 339 intravenous catheter insertions. Heart Lung. 2005[cited 2019 Feb 21]; 34(5): 345-59. DOI: https://doi.org/10.1016/j.hrtlng.2005.04.002

22. Prunet B, Meaudre E, Montcriol A, Asencio Y, Bordes J, Lacroix G. A prospective randomized trial of two safety peripheral intravenous catheters. Anesth. Analg. 2008 [cited 2019 Feb 21]; 107(1):155-8. DOI: https://doi.org/10.1213/ane.0b013e318174df5f

23. Malta DC, Bernal RTI, Lima MG, Araújo SSC, Silva MMA, Freitas MIF, et al. Noncommunicable diseases and the use of health services: analysis of the National Health Survey in Brazil. Rev. Saude Publica. 2017[cited 2019 Feb 17]; 51(Supl 1:4s). DOI: https://doi.org/10.1590/S1518-8787.2017051000090

24. Pagnutti L, Bin A, Donato R, Di Lena G, Fabbro C, Fornasiero L et al. Difficult intravenous access tool in patients receiving peripheral chemotherapy: a pilot-validation study. J. Oncol. Nurs. 2016 [cited 2019 Feb 21]: 58-63. DOI: https://doi.org/10.1016/j.ejon.2015.06.008

25. Oliveira AM, Danski MTR, Oliveira EP. Ultrasound-guided peripheral venipuncture: prevalence of success and associated factors. Cogitare Enferm. 2017 [cited 2019 Feb 21]; (22)3: e49599. DOI: http://dx.doi.org/10.5380/ce.v22i3.49599

26. Campos LB, Martins JR, Arreguy-Sena C, Alves MS, Teixeira CV, Souza LC. Experiences of hospitalized patients with the venipuncture process. Esc. Anna Nery Rev. Enferm. 2016 [cited 2019 Feb 18]; 20(3): e20160078. DOI: http://dx.doi.org/10.5935/1414-8145.20160078

27. Alves DA, Lucas TC, Martins DA, Cristianismo RS, Braga EVO, Guedes HM. Evaluation of peripheral intravenous cateter puncture and maintenance procedures. RECOM. 2019 [cited 2019 Feb 12]; 9: e3005. DOI: http://dx.doi.org/10.19175/recom.v9i0.3005

28. Pereira KC, Cogo ALP, Silva APSS Critical analysis of peripheral catheter venipuncture videos available on YouTube. REME rev. min. enferm. 2016 [cited 2019 Feb 18]; 20:e970. Available from: http://www.reme.org.br/exportar-pdf/1106/e970.pdf

29. Oliveira FT, Machado KMF, Santos LPR, Quiroz NR, Silva J, et al. Praxis of nursing students in intravenous therapy: a before and after study. Rev. enferm. UERJ. 2014 [cited 2019 Aug 25]; 22(2):219-25. Available from: http://www.facenf.uerj.br/v22n2/v22n2a12.pdf

30. Costa LM, Benet ERR, Pai DD, Camponogara S. Factors that influence the use of peripherally inserted central catheter in adult patients Rev. enferm. UERJ.2017 [cited 2019 Aug 28]; 25:e20976. DOI: http://dx.doi.org/10.12957/reuerj.2017.20976 